\title{
Safety of inhaled glycopyrronium in patients with COPD: a comprehensive analysis of clinical studies and post-marketing data
}

This article was published in the following Dove Press journal:

International Journal of COPD

II August 2015

Number of times this article has been viewed

\author{
Anthony D D'Urzo' \\ Edward M Kerwin ${ }^{2}$ \\ Kenneth R Chapman ${ }^{3}$ \\ Marc Decramer ${ }^{4}$ \\ Robert DiGiovanni ${ }^{5}$ \\ Peter D'Andrea ${ }^{6}$ \\ Huilin $\mathrm{Hu}^{6}$ \\ Pankaj Goyal ${ }^{5}$ \\ Pablo Altman ${ }^{6}$ \\ 'Department of Family and \\ Community Medicine, University \\ of Toronto, Toronto, ON, Canada; \\ ${ }^{2}$ Clinical Research Institute of \\ Southern Oregon, PC, Medford, \\ USA; ${ }^{3}$ Asthma and Airway Centre, \\ University Health Network, Toronto \\ Western Hospital, Toronto, ON, \\ Canada; ${ }^{4}$ Respiratory Division, \\ University of Leuven, Leuven, \\ Belgium; ${ }^{5}$ Novartis Pharma AG, \\ Basel, Switzerland; ${ }^{6}$ Novartis \\ Pharmaceuticals Corporation, East \\ Hanover, NJ, USA
}

Background: Chronic use of inhaled anticholinergics by patients with chronic obstructive pulmonary disease (COPD) has raised long-term safety concerns, particularly cardiovascular. Glycopyrronium is a once-daily anticholinergic with greater receptor selectivity than previously available agents.

Methods: We assessed the safety of inhaled glycopyrronium using data pooled from two analysis sets, involving six clinical studies and over 4,000 patients with COPD who received one of the following treatments: glycopyrronium $50 \mu \mathrm{g}$, placebo (both delivered via the Breezhaler ${ }^{\circledR}$ device), or tiotropium $18 \mu \mathrm{g}$ (delivered via the HandiHaler ${ }^{\circledR}$ device). Data were pooled from studies that varied in their duration and severity of COPD of the patients (ie, $\leq 12$ weeks duration with patients having moderate or severe COPD; and $>1$ year duration with patients having severe and very severe COPD). Safety comparisons were made for glycopyrronium vs tiotropium or placebo. Poisson regression was used to assess the relative risk for either active drug or placebo (and between drugs where placebo was not available) for assessing the incidence of safety events. During post-marketing surveillance (PMS), safety was assessed by obtaining reports from various sources, and disproportionality scores were computed using EMPIRICA ${ }^{\mathrm{TM}}$. In particular, the cardiac safety of glycopyrronium during the post-marketing phase was evaluated.

Results: The overall incidence of adverse events and deaths was similar across groups, while the incidence of serious adverse events was numerically higher in placebo. Furthermore, glycopyrronium did not result in an increased risk of cerebro-cardiovascular events vs placebo. There were no new safety reports during the PMS phase that suggested an increased risk compared to results from the clinical studies. Moreover, the cardiac safety of glycopyrronium during the PMS phase was also consistent with the clinical data.

Conclusion: The overall safety profile of glycopyrronium was similar to its comparators indicating no increase in the overall risk for any of the investigated safety end points.

Keywords: COPD, drug safety, glycopyrronium, post-marketing surveillance

\section{Introduction}

Chronic obstructive pulmonary disease (COPD) is characterized by airflow limitation that is not only progressive but also irreversible. ${ }^{1}$ Inhaled bronchodilators are regarded as foundational pharmacologic agents in the management of patients with COPD, providing not only short-term symptom relief but also reducing the exacerbation frequency and improving the quality of life. ${ }^{1}$ Long-acting muscarinic antagonists (LAMAs) (either alone or in combination with $\beta$-agonists) have been found to be effective bronchodilators for the treatment of patients with COPD. ${ }^{1}$ LAMAs may produce typical anticholinergic side effects such as dry mouth, urinary retention, constipation, and nausea. ${ }^{2}$ Inhaled tiotropium administered via soft mist inhaler at moderate and high dosages has been
Correspondence: Anthony D D'Urzo Department of Family and Community Medicine, University of Toronto, 1670 Dufferin Street, Suite 107, Toronto, ON M6H3M2, Canada

Tel + I 4l 66529336

Fax + | 4| 66520218

Email tonydurzo@sympatico.ca 
associated with the risk of increased mortality, in particular, cardiovascular mortality. ${ }^{3}$ However, recent trials, such as the Tiotropium Safety and Performance in Respimat (TIOSPIR) study $^{4}$ and other more recent analyses show a mortality risk similar to that of tiotropium in dry powder $\left(\right.$ HandiHaler $\left.^{\circledR}\right)$ formulation. ${ }^{5-9}$ Glycopyrronium $(50 \mu \mathrm{g})$, delivered via the Breezhaler $^{\circledR}$ device, is a once-daily (od) LAMA that is indicated for management of patients with COPD. Its efficacy and safety have been demonstrated in various clinical studies. ${ }^{10}$ It is known to be safe and well tolerated while exhibiting sustained 24-hour bronchodilation in patients with moderateto-severe COPD. ${ }^{11}$ As compared to tiotropium, it has a greater selectivity for the M3 vs M2 receptor, a property that may reduce the risk of cardiovascular adverse events (AEs). ${ }^{12}$ Additionally, it has been shown to exhibit a rapid onset of action as compared to tiotropium, thus allowing for rapid and sustained symptom relief. ${ }^{11}$ To assess the safety of glycopyrronium, we have undertaken an analysis of pooled safety data from clinical trials involving glycopyrronium along with the available data from post-marketing surveillance (PMS) review periods. In this pooled analysis, the safety of glycopyrronium (delivered via the Breezhaler ${ }^{\circledR}$ device) and tiotropium (delivered via the HandiHaler ${ }^{\circledR}$ device) are compared with that of placebo. In the absence of placebo as a comparator, glycopyrronium is also compared with tiotropium.

\section{Methods}

All patients provided written informed consent. The comprehensive evaluation of the safety of glycopyrronium in the clinical studies was performed by using two distinct analysis sets that comprise the COPD core safety database $(\mathrm{S}-\mathrm{db})$ and the COPD long-term S-db. Studies with the recommended regimen of $50 \mu \mathrm{g}$ glycopyrronium od, that were randomized double-blinded, placebo- and/or active-controlled, parallel design, with exposure duration of at least 12 weeks in patients with moderate or severe COPD (including following studies: CNVA237A2304 [GLOW1], ${ }^{13}$ CNVA237A2303 [GLOW2], ${ }^{14}$ CQVA149A2303 [SHINE], ${ }^{15}$ CNVA237A2314 [GLOW5], ${ }^{16}$ and CNVA237A2309 [GLOW7]) ${ }^{17}$ were included in the COPD core S-db. Within this database, data were pooled from all studies with similar disease severity, study design, and assessment methods utilized for evaluating AEs, deaths, and events of interest, with results adjusted for the length of exposure and reported as incidence (number of events per 100 patient treatment years [PTYs, sum of the duration of exposure over patients, in days/365.25 days]). The COPD long-term S-db included studies with an exposure duration of $>1$ year (CQVA149A2304 [SPARK], ${ }^{18}$ a doubleblinded, active-controlled, parallel design, and an exposure duration of at least 15 months in patients with severe and very severe COPD). The design of six individual studies included in the present analysis is summarized in Table 1. All the major adverse cardiovascular events and deaths were adjudicated by an external committee using predefined criteria. For the analysis of safety during the PMS review period, patient exposure to glycopyrronium was estimated based on the cumulative worldwide sales volume since its availability (September 28, 2012) for the approved $50 \mu \mathrm{g}$

Table I Details of studies included in the pooled analysis

\begin{tabular}{|c|c|c|c|c|c|c|}
\hline Study name & $\begin{array}{l}\text { Study } \\
\text { design }\end{array}$ & $\begin{array}{l}\text { Study } \\
\text { duration }\end{array}$ & $\begin{array}{l}\text { Patients } \\
\text { randomized }(\mathrm{N})\end{array}$ & Patient population & Treatment groups & References \\
\hline $\begin{array}{l}\text { GLOWI } \\
\text { (CNVA237A2304) }\end{array}$ & $R, D B, P C, P G$ & 26 weeks & 822 & $\begin{array}{l}\text { Moderate-to-severe COPD } \\
\text { (Stage II and III, GOLD 2008) }\end{array}$ & $\begin{array}{l}\text { GLY } 50 \mu g \text { od } \\
\text { PBO od }\end{array}$ & 13 \\
\hline $\begin{array}{l}\text { GLOW2 } \\
\text { (CNVA237A2303) }\end{array}$ & $\begin{array}{l}\text { R, DB, and } \\
\text { OL (TIO), } \\
\text { PC, PG }\end{array}$ & 52 weeks & 1,066 & $\begin{array}{l}\text { Moderate-to-severe COPD } \\
\text { (Stage II and III, GOLD 2008) }\end{array}$ & $\begin{array}{l}\text { GLY } 50 \mu g \text { od } \\
\text { PBO od } \\
\text { OL-TIO I } 8 \mu g \text { od }\end{array}$ & 14 \\
\hline $\begin{array}{l}\text { GLOW5 } \\
\text { (CNVA237A23|4) }\end{array}$ & $\begin{array}{l}\text { R, DD, PG, } \\
\mathrm{BL}(\mathrm{TIO})\end{array}$ & 12 weeks & 657 & $\begin{array}{l}\text { Moderate-to-severe COPD } \\
\text { (Stage II and III, GOLD 20I0) }\end{array}$ & $\begin{array}{l}\text { GLY } 50 \mu \mathrm{g} \text { od } \\
\text { Blinded TIO } 18 \mu \mathrm{g} \text { od }\end{array}$ & 16 \\
\hline $\begin{array}{l}\text { GLOW7 } \\
\text { (CNVA237A2309) }\end{array}$ & $\begin{array}{l}\text { R, DB, PC, } \\
P G\end{array}$ & 26 weeks & 459 & $\begin{array}{l}\text { Moderate-to-severe COPD } \\
\text { (Stage II and III, GOLD 20I0) }\end{array}$ & $\begin{array}{l}\text { GLY } 50 \mu g \text { od } \\
\text { PBO od }\end{array}$ & 17 \\
\hline $\begin{array}{l}\text { SHINE* } \\
\text { (CQVAI49A2303) }\end{array}$ & $\begin{array}{l}\mathrm{DB} \text { and } \mathrm{OL} \\
(\mathrm{TIO}), \mathrm{PG}, \mathrm{R}, \\
\mathrm{PC}, \mathrm{AC}\end{array}$ & 26 weeks & 2,144 & $\begin{array}{l}\text { Moderate-to-severe COPD } \\
\text { (Stage II and III, GOLD 2008) }\end{array}$ & $\begin{array}{l}\text { QVAI } 49 \text { I IO/50 } \mu \mathrm{g} \text { od } \\
\text { IND I } 50 \mu \mathrm{g} \text { od } \\
\text { GLY } 50 \mu \mathrm{g} \text { od } \\
\text { OL-TIO I8 } 4 \mathrm{~g} \text { od } \\
\text { PBO od }\end{array}$ & 15 \\
\hline $\begin{array}{l}\text { SPARK* } \\
\text { (CQVAI49A2304) }\end{array}$ & $\begin{array}{l}\mathrm{DB} \text { and } \mathrm{OL} \\
\text { (TIO), PG, R }\end{array}$ & 64 weeks & 2,224 & $\begin{array}{l}\text { Severe-to-very severe COPD } \\
\text { (Stage III and IV, GOLD 2008) }\end{array}$ & $\begin{array}{l}\text { QVAI } 49 \text { I I } 0 / 50 \mu \mathrm{g} \text { od } \\
\text { GLY } 50 \mu \mathrm{g} \text { od } \\
\text { OL-TIO I } 8 \mu \mathrm{g} \text { od }\end{array}$ & 18 \\
\hline
\end{tabular}

Note: *Safety data for IND and QVAI49 arms from the SHINE study, and the QVAI49 arm from the SPARK study was not included in the current analysis.

Abbreviations: AC, active controlled; BL, blinded; COPD, chronic obstructive pulmonary disease; DB, double-blind; DD, double-dummy; GLOW, GLycopyrronium bromide in COPD airWays clinical study; GLY, glycopyrronium; IND, indacaterol; od, once daily; OL, open-label; PBO, placebo; PC, placebo-controlled; PG, parallel-group; R, randomized; TIO, tiotropium. 
dose delivered via the Breezhaler ${ }^{\circledR}$ device, until the cut-off date of March 28, 2014.

\section{Patients}

The protocols for all studies were approved by institutional review boards and ethics committees at participating centers, and were conducted in accordance with the Declaration of Helsinki and Good Clinical Practice. All patients provided written informed consent. Overall, the inclusion and exclusion criteria for patients were similar across the studies. The study population in all the trials comprised patients who were at least 40 years of age, had a smoking history of at least 10 pack-years, with post-bronchodilator forced expiratory volume in 1 second $\left(\mathrm{FEV}_{1}\right)$ to forced vital capacity (FVC) ratio of $<0.70$ at screening, and diagnosed with moderate-to-severe COPD (Stage II or III according to the GOLD 2005 and 2008 criteria; post-bronchodilator $\mathrm{FEV}_{1}$ of $\geq 30 \%$ and $<80 \%$ of the predicted normal) with the exception of the SPARK study that enrolled patients with severeto-very severe COPD (Stage IV according to GOLD 2008; post-bronchodilator $\mathrm{FEV}_{1} \leq 50 \%$ predicted). ${ }^{18}$ Additionally, the clinical trial population also comprised patients with a medical history of stable cardiovascular disease.

\section{Safety assessment}

All analyses were based on the safety population, defined as all patients who received at least one dose of study medication. Common AEs were summarized according to the Medical Dictionary for Regulatory Activities (MedDRA, version 16.0) hierarchy, including primary system organ class and preferred term. Only those events regarded as identified risks (narrow-angle glaucoma, bladder outflow obstruction and urinary retention, and use in patients with severe renal impairment) and potential risks (cerebrovascular events, cardiovascular events including myocardial infarction and cardiac arrhythmias, atrial fibrillation, paradoxical bronchospasm, and medication errors) in the glycopyrronium (Seebri ${ }^{\circledR}$ Breezhaler $^{\circledR}$ ) risk management plan were searched through Standardized MedDRA Queries (SMQs) and Novartis MedDRA queries (NMQs). SMQs are collections of MedDRA (preferred) terms that relate to a defined medical condition, and allow identification of safety concerns, whereas NMQs are a customized group of search terms that define a medical concept for which there is no SMQ available.

The safety profile of glycopyrronium during the PMS review period was assessed by deriving AEs obtained from individual case study reports from health care professionals, consumers, scientific literature, spontaneous reports, competent authorities, noninterventional studies, and solicited sources such as compassionate use programs.

Furthermore, the safety of glycopyrronium during the PMS review period was also assessed by recording spontaneous voluntary reports of AEs. The EMPIRICA ${ }^{\mathrm{TM}}$ Signal System was used to calculate the disproportionality scores of the reported AEs using the Multi-item Gamma Poisson Shrinker (MGPS) algorithm. EMPIRICA ${ }^{\mathrm{TM}}$ is an advanced data mining tool used for automated detection and quantification of safety signals, applied to ARGUS ${ }^{\text {TM }}$ (a Novartis global S-db that provides a comprehensive AE management system to support the Novartis pharmacovigilance program). The safety data coded by MedDRA (version 16.0) against events marked as "diagnosis" in ARGUSTM (leading events only) were retrieved by EMPIRICA ${ }^{\mathrm{TM}}$, after which all the AEs were assigned a statistical score.

\section{Statistical analysis}

For each event of interest from the clinical data, an estimate of the risk ratio (RR) of incidence density (each respective active treatment vs placebo or active control), together with the $95 \%$ confidence interval (CI), was produced through a Poisson regression with treatment and study as class effects in the model.

For the assessment of safety during the PMS review period, the statistical scores (represented as Empirical Bayes Geometric Mean [EBGM]) were calculated using the MGPS algorithm. The assignment of EBGM scores was based on the association of glycopyrronium and AEs together (drugevent combination) with a disproportionately high occurrence of reports, as compared to the proportion of reports in the entire ARGUS ${ }^{\mathrm{TM}}$ database for glycopyrronium and AEs independently. The lower $90 \%$ CI limit of EBGM (denoted as EB05) was used.

EB05 values $<2$ were considered as no technical signal and EMPIRICA ${ }^{\mathrm{TM}}$ alerted the reviewers if the reporting proportions of AEs were above the threshold value. ${ }^{19}$ EMPIRICA $^{\mathrm{TM}}$ calculates the disproportionality scores afresh each time to account for reconciliation of historic safety data. The disproportionality scores presented in this analysis were calculated on July 15, 2014.

\section{Results \\ Patients and duration of their exposure to treatment}

The analysis of the COPD core S-db included data from 4,178 patients. The length of time for which the patients were exposed to the study drugs is tabulated in Table 2 . 
Table 2 Duration of exposure to study drug after randomization (COPD core S-db)

\begin{tabular}{|c|c|c|c|}
\hline Duration of exposure & $\begin{array}{l}\text { GLY } 50 \mu \mathrm{g} \\
\mathrm{N}=2,180\end{array}$ & $\begin{array}{l}\text { TIO } 18 \mu \mathrm{g} \\
\mathrm{N}=1,077\end{array}$ & $\begin{array}{l}\text { PBO } \\
\mathrm{N}=92 \text { I }\end{array}$ \\
\hline Total PTYs & $\mathrm{I}, 138.642$ & 534.234 & 508.216 \\
\hline \multicolumn{4}{|c|}{ PTYs exposure, $\%$ of patients (Total PTYs) } \\
\hline I day & $0.4 \mathrm{I}(0.025)$ & $0.37(0.011)$ & $0.54(0.014)$ \\
\hline 2 days $-<2$ weeks & $1.38(0.605)$ & I.II (0.162) & $2.39(0.408)$ \\
\hline 2 weeks $-<1$ month & $1.83(2.502)$ & $1.67(1.112)$ & $4.13(2.042)$ \\
\hline I month $-<3$ months & 18.62 (86.439) & 30.64 (74.209) & $6.5 \mathrm{I}(9.785)$ \\
\hline 3 months $-<6$ months & 12.25 (I I5.269) & $10.68(49.109)$ & $12.49(51.425)$ \\
\hline 6 months $-<12$ months & $52.34(645.478)$ & $4 I .13(253.74 I)$ & $58.09(297.736)$ \\
\hline$\geq 12$ months & 13.17 (288.323) & $14.39(155.890)$ & $15.85(146.806)$ \\
\hline
\end{tabular}

Abbreviations: COPD, chronic obstructive pulmonary disease; GLY, glycopyrronium; N, patients randomized; PBO, placebo; PTYs, patient treatment years; S-db, safety database; TIO, tiotropium.

The majority of PTYs occurred in patients treated from $3->12$ months on these studies. The characteristics of the pooled patient population along with other clinical characteristics were comparable across all the treatment groups (Table 3). There were a few noteworthy differences in the glycopyrronium group vs the other treatment groups: 1) by virtue of the way the studies were designed, the glycopyrronium group exhibited approximately twofold higher patient exposure and enrollment than the tiotropium and placebo groups (Table 2); 2 ) more patients were $\geq 85$ years $(0.41 \%$, $0.19 \%$, and $0.22 \%$, for glycopyrronium, tiotropium, and placebo groups, respectively) (Table 3 ).

\section{Patient exposure during PMS review period}

The cumulative worldwide sale of glycopyrronium (at its approved dosage of $50 \mu \mathrm{g}$ ) during the PMS review period is estimated to be approximately $3,332 \mathrm{~g}$ of the active pharmaceutical ingredient. Consequently, patient exposure to glycopyrronium (since its first availability and until the cut-off date) is estimated to be approximately 182,562 PTYs.

\section{AEs during clinical studies}

The five most common AEs in the glycopyrronium group were COPD worsening, nasopharyngitis, upper respiratory tract infection, cough, and headache (Table 4). Across all groups, COPD worsening was the most common $\mathrm{AE}$ that exhibited the least incidence in the glycopyrronium group. Generally, AEs were well balanced and the rates of occurrence of events for both glycopyrronium and tiotropium treatment arms were similar to that of placebo. The only AEs (among the five most common AEs) observed in $>2 \%$ of patients and that numerically increased with glycopyrronium were nasopharyngitis and headache, which occurred with an incidence similar to that of placebo and tiotropium.
Overall, the cardiovascular AE rate was similar for glycopyrronium and placebo although atrial fibrillation was seen more often with glycopyrronium. The most commonly occurring LAMA-specific AEs were dizziness, dry mouth, constipation, nausea, and pyrexia. In general, these events

Table 3 Characteristics of pooled clinical trial study population (COPD core S-db)

\begin{tabular}{|c|c|c|c|}
\hline $\begin{array}{l}\text { Patient } \\
\text { characteristics }\end{array}$ & $\begin{array}{l}\text { GLY } 50 \mu \mathrm{g} \\
\mathbf{N}=\mathbf{2}, 180\end{array}$ & $\begin{array}{l}\text { TIO } 18 \mu \mathrm{g} \\
\mathrm{N}=1,077\end{array}$ & $\begin{array}{l}\text { PBO } \\
\mathrm{N}=921\end{array}$ \\
\hline \multicolumn{4}{|l|}{ Age } \\
\hline$<65$ years & $\mathrm{I}, \mathrm{I} 28(5 \mathrm{I} .74)$ & $575(53.39)$ & $455(49.40)$ \\
\hline $65-<75$ years & $792(36.33)$ & $387(35.93)$ & $350(38.00)$ \\
\hline $75-<85$ years & $25 I(1 I .5 I)$ & $113(10.49)$ & $114(12.38)$ \\
\hline$\geq 85$ years & $9(0.4 I)$ & $2(0.19)$ & $2(0.22)$ \\
\hline \multicolumn{4}{|l|}{ Sex } \\
\hline Male & I,689 (77.48) & $776(72.05)$ & $702(76.22)$ \\
\hline Female & $491(22.52)$ & $30 I(27.95)$ & $219(23.78)$ \\
\hline \multicolumn{4}{|l|}{ Race } \\
\hline Caucasian & $\mathrm{I}, 345(6 \mathrm{I} .70)$ & $786(72.98)$ & $557(60.48)$ \\
\hline Black & $28(1.28)$ & $17(1.58)$ & $15(1.63)$ \\
\hline Asian & $758(34.77)$ & $24 I(22.38)$ & $331(35.94)$ \\
\hline Other & $49(2.25)$ & $33(3.06)$ & $18(1.95)$ \\
\hline \multicolumn{4}{|c|}{ Number of CCV risk factors at baseline } \\
\hline 0 & $217(9.95)$ & $79(7.34)$ & $90(9.77)$ \\
\hline I & $571(26.19)$ & $255(23.68)$ & $236(25.62)$ \\
\hline 2 & $570(26.15)$ & $286(26.56)$ & $243(26.38)$ \\
\hline$\geq 3$ & $822(37.7 I)$ & $457(42.43)$ & $352(38.22)$ \\
\hline \multicolumn{4}{|l|}{ COPD severity } \\
\hline Mild & $2(0.09)$ & I (0.09) & $0(0)$ \\
\hline Moderate & I,302 (59.72) & $66 \mid(61.37)$ & $579(62.87)$ \\
\hline Severe & $868(39.82)$ & $414(38.44)$ & $338(36.70)$ \\
\hline Very severe & $8(0.37)$ & $0(0)$ & $4(0.43)$ \\
\hline \multicolumn{4}{|l|}{ Steroid use } \\
\hline None & 948 (43.49) & $48 I(44.66)$ & $427(46.36)$ \\
\hline ICS & $\mathrm{I}, 220(55.96)$ & $588(54.60)$ & 491 (53.3I) \\
\hline OCS & $3(0.14)$ & $2(0.19)$ & I (0.II) \\
\hline ICS and OCS & $9(0.4 I)$ & $6(0.56)$ & $2(0.22)$ \\
\hline $\begin{array}{l}\text { Baseline diabetes } \\
\text { condition }\end{array}$ & $274(12.57)$ & 141 (13.09) & $119(12.92)$ \\
\hline
\end{tabular}

Note: Values are $\mathrm{n}(\%)$.

Abbreviations: CCV, cerebrovascular and cardiovascular; COPD, chronic obstructive pulmonary disease; GLY, glycopyrronium; ICS, inhaled corticosteroids; N, patients randomized; OCS, oral corticosteroids; PBO, placebo; S-db, safety database; TIO, tiotropium. 
Table 4 Incidence of most common AEs (per 100 PTYs) in clinical studies sorted by primary system organ class and preferred term ( $>10$ events/I00 PTYs for GLY) (COPD core S-db)

\begin{tabular}{|c|c|c|c|}
\hline $\begin{array}{l}\text { Primary system organ class, } \\
\text { preferred term }\end{array}$ & $\begin{array}{l}\text { GLY } 50 \mu \mathrm{g} \\
\mathrm{N}=2,180\end{array}$ & $\begin{array}{l}\text { TIO } 18 \mu \mathrm{g} \\
\mathrm{N}=1,077\end{array}$ & $\begin{array}{l}\text { PBO } \\
\mathrm{N}=921\end{array}$ \\
\hline Patients with $\geq \mathrm{I} A E,(\%)$ & I,274 (58.44) & $607(56.36)$ & $586(63.63)$ \\
\hline Number of AEs/I00 PTYs & 342.952 & 371.373 & 393.927 \\
\hline \multicolumn{4}{|c|}{ Respiratory, thoracic, and mediastinal disorders } \\
\hline Total & $\mathrm{I}, 22 \mathrm{I}(107.233)$ & $682(127.659)$ & 709 (139.508) \\
\hline COPD worsening & $868(76.231)$ & $510(95.464)$ & $538(105.860)$ \\
\hline Cough & $87(7.64 I)$ & $39(7.300)$ & $39(7.674)$ \\
\hline Dyspnea & $53(4.655)$ & $19(3.556)$ & $29(5.706)$ \\
\hline Oropharyngeal pain & $27(2.37 \mathrm{I})$ & I4 (2.62I) & $16(3.148)$ \\
\hline Sinus congestion & $16(1.405)$ & $5(0.936)$ & $2(0.394)$ \\
\hline Dysphonia & $14(1.230)$ & $7(1.310)$ & $5(0.984)$ \\
\hline Nasal congestion & $12(1.054)$ & $8(1.497)$ & $8(1.574)$ \\
\hline Epistaxis & II (0.966) & $5(0.936)$ & $2(0.394)$ \\
\hline \multicolumn{4}{|l|}{ Infections and infestations } \\
\hline Total & $\mathrm{I}, 029(90.37 \mathrm{I})$ & $546(102.202)$ & $537(105.664)$ \\
\hline Nasopharyngitis & $209(18.355)$ & 79 (14.788) & 93 (I8.299) \\
\hline Upper RTI & $170(14.930)$ & $73(13.664)$ & $100(19.677)$ \\
\hline Lower RTI & $55(4.830)$ & $34(6.364)$ & $28(5.509)$ \\
\hline Bronchitis & $43(3.776)$ & $29(5.428)$ & $24(4.722)$ \\
\hline Sinusitis & $42(3.689)$ & $22(4.118)$ & $20(3.935)$ \\
\hline Urinary tract infection & $42(3.689)$ & $23(4.305)$ & I $3(2.558)$ \\
\hline Viral upper RTI & $42(3.689)$ & $35(6.55 \mathrm{I})$ & 38 (7.477) \\
\hline Influenza & $34(2.986)$ & $18(3.369)$ & I 3 (2.558) \\
\hline Pneumonia & $31(2.723)$ & $16(2.995)$ & 22 (4.329) \\
\hline Pharyngitis & $20(1.756)$ & $17(3.182)$ & $8(1.574)$ \\
\hline Rhinitis & $19(1.669)$ & $7(1.310)$ & $4(0.787)$ \\
\hline Cellulitis & $12(1.054)$ & $6(1.123)$ & $6(1.18 I)$ \\
\hline Oral candidiasis & $12(1.054)$ & $7(1.310)$ & $6(1.181)$ \\
\hline Gastroenteritis & II (0.966) & $3(0.562)$ & $6(1.18 I)$ \\
\hline Gastroenteritis viral & $10(0.878)$ & $4(0.749)$ & $3(0.590)$ \\
\hline RTI & $10(0.878)$ & $5(0.936)$ & $3(0.590)$ \\
\hline \multicolumn{4}{|l|}{ Nervous system disorders } \\
\hline Total & $205(18.004)$ & $82(15.349)$ & $83(16.332)$ \\
\hline Headache & $82(7.202)$ & $38(7.113)$ & $33(6.493)$ \\
\hline Syncope & $14(1.230)$ & 0 & $4(0.787)$ \\
\hline \multicolumn{4}{|l|}{ Cardiac disorders } \\
\hline Total & $104(9.134)$ & $34(6.364)$ & $55(10.822)$ \\
\hline Atrial fibrillation & $15(1.317)$ & $4(0.749)$ & $2(0.394)$ \\
\hline Angina pectoris & $10(0.878)$ & $5(0.936)$ & $10(1.968)$ \\
\hline \multicolumn{4}{|l|}{ Eye disorders } \\
\hline Total & $43(3.776)$ & $23(4.305)$ & 19 (3.739) \\
\hline Cataract & $10(0.878)$ & $7(1.310)$ & $2(0.394)$ \\
\hline \multicolumn{4}{|l|}{ Gastrointestinal disorders } \\
\hline Total & $263(23.098)$ & 140 (26.206) & I 38 (27.154) \\
\hline Diarrhea & $29(2.547)$ & $10(1.872)$ & $18(3.542)$ \\
\hline Toothache & $16(1.405)$ & $5(0.936)$ & $4(0.787)$ \\
\hline Dyspepsia & $15(1.317)$ & $6(1.123)$ & $4(0.787)$ \\
\hline Abdominal pain & $13(1.142)$ & $8(1.497)$ & $5(0.984)$ \\
\hline Vomiting & $13(1.142)$ & $10(1.872)$ & II (2.164) \\
\hline Gastroesophageal reflux disease & $12(1.054)$ & $9(1.685)$ & $10(1.968)$ \\
\hline
\end{tabular}

Note: Values are total number of AEs (AEs/I00 PTYs) unless otherwise stated.

Abbreviations: AEs, adverse events; COPD, chronic obstructive pulmonary disease; GLY, glycopyrronium; N, patients randomized; PBO, placebo; PTYs, patient treatment years; RTI, respiratory tract infection; S-db, safety database; TIO, tiotropium.

occurred most commonly in the placebo group, with only dry mouth, constipation, and throat irritation modestly increasing with glycopyrronium (Table 5).

\section{Adjudicated deaths and serious AEs reported during clinical phase}

The incidence of deaths and serious AEs (SAEs) adjusted per PTYs is listed in Table 6. The occurrence of deaths (exposure adjusted) was comparable across groups. Respiratory cause was the leading reason for deaths among the treatment groups, and was lowest in the glycopyrronium group. Furthermore, the RR for respiratory deaths relative to placebo was slightly lower for glycopyrronium (RR: 1.059 vs placebo; 95\% $\mathrm{CI}-0.368,3.047)$ than for tiotropium (RR: 1.928 vs placebo; 95\% CI - 0.471, 7.892) (Table 6). The overall incidence of SAEs (exposure adjusted) in glycopyrronium and tiotropium 
Table 5 Incidence of AEs most commonly associated with LAMAs, classified according to primary system organ class and preferred term (COPD core S-db)

\begin{tabular}{|c|c|c|c|}
\hline $\begin{array}{l}\text { Primary system organ } \\
\text { class, preferred term }\end{array}$ & $\begin{array}{l}\text { GLY } 50 \mu \mathrm{g} \\
\mathrm{N}=2,180\end{array}$ & $\begin{array}{l}\text { TIO } 18 \mu \mathrm{g} \\
\mathrm{N}=1,077\end{array}$ & $\begin{array}{l}\mathrm{PBO} \\
\mathrm{N}=921\end{array}$ \\
\hline Total PTYs & $\mathrm{I}, \mathrm{I} 38.642$ & 534.234 & 508.216 \\
\hline \multicolumn{4}{|l|}{ Nervous system disorders } \\
\hline Dizziness & $25(2.196)$ & $9(1.685)$ & I $3(2.558)$ \\
\hline \multicolumn{4}{|l|}{ Renal and urinary disorders } \\
\hline Urinary retention & $4(0.35 I)$ & $2(0.374)$ & 0 \\
\hline \multicolumn{4}{|l|}{ Gastrointestinal disorders } \\
\hline Dry mouth & $33(2.898)$ & $14(2.621)$ & $10(1.968)$ \\
\hline Constipation & $22(1.932)$ & $5(0.936)$ & $9(1.77 I)$ \\
\hline Nausea & $16(1.405)$ & II (2.059) & II (2.164) \\
\hline \multicolumn{4}{|l|}{ General disorders } \\
\hline Pyrexia & $35(3.074)$ & $9(1.685)$ & 25 (4.919) \\
\hline \multicolumn{4}{|l|}{ Eye disorders } \\
\hline Vision blurred & $5(0.439)$ & I (0.187) & $3(0.590)$ \\
\hline Dry eye & $3(0.263)$ & $2(0.374)$ & I (0.197) \\
\hline \multicolumn{4}{|l|}{ Respiratory disorders } \\
\hline Throat irritation & II (0.966) & $3(0.562)$ & $4(0.787)$ \\
\hline Rhinorrhea & $5(0.439)$ & $6(1.123)$ & $10(1.968)$ \\
\hline \multicolumn{4}{|l|}{ Cardiac disorders } \\
\hline Tachycardia & $2(0.176)$ & I (0.187) & $4(0.787)$ \\
\hline Palpitations & $5(0.439)$ & $2(0.374)$ & $4(0.787)$ \\
\hline
\end{tabular}

Note: Values are total number of AEs (AEs/I00 PTYs).

Abbreviations: AEs, adverse events; COPD, chronic obstructive pulmonary disease; GLY, glycopyrronium; LAMAs, long-acting muscarinic antagonists; N, patients randomized; PBO, placebo; PTYs, patient treatment years; S-db, safety database; TIO, tiotropium.

groups was lower than that in the placebo group. The most commonly occurring SAE was COPD worsening, which was the lowest in the glycopyrronium group.

The incidence of SAEs by preferred term for gastrointestinal, vascular, and renal/urinary disorders was lower than the threshold ( $>0.2$ events/100 PTYs for glycopyrronium). The overall frequency of occurrence (events/100 PTYs) of SAEs in the various treatment arms was as follows: glycopyrronium (gastrointestinal, 1.317; vascular, 0.790; renal/urinary, 0.439); tiotropium (gastrointestinal, 1.872; vascular, 0; renal/urinary, 0.187); and placebo (gastrointestinal, 2.558; vascular, 0.394; renal/urinary, 0.787).

\section{Cerebrovascular and cardiovascular events during clinical phase}

The frequency of occurrence of cerebrovascular and cardiovascular (CCV) events are shown in Table 7 (also refer Supplementary materials, Tables S1-S4). Glycopyrronium did not result in any increased risk for cardiovascular events in patients in comparison with placebo. Cumulative assessment of the clinical data did not establish any underlying association of cardiovascular events and glycopyrronium. Compared to placebo, both actives, glycopyrronium (RR:
Table 6 Adjudicated deaths, and SAEs (>0.2 events/100 PTYs for GLY) (COPD core S-db)

\begin{tabular}{|c|c|c|c|}
\hline $\begin{array}{l}\text { Primary system organ } \\
\text { class, preferred term }\end{array}$ & $\begin{array}{l}\text { GLY } 50 \mu \mathrm{g} \\
\mathrm{N}=2,180\end{array}$ & $\begin{array}{l}\text { TIO } 18 \mu \mathrm{g} \\
\mathrm{N}=1,077\end{array}$ & $\begin{array}{l}\text { PBO } \\
\mathrm{N}=921\end{array}$ \\
\hline Total deaths, n (\%) & II (0.23) & $5(0.46)$ & $5(0.54)$ \\
\hline Risk ratio & 1.059 & 1.928 & \\
\hline $95 \% \mathrm{Cl}$ (lower, upper limit) & $0.368,3.047$ & $0.471,7.892$ & \\
\hline$P$-value & 0.9160 & 0.3610 & \\
\hline Deaths/I00 PTYs & 0.966 & 0.936 & 0.984 \\
\hline Cardiovascular & 0.263 & 0.187 & 0.197 \\
\hline Respiratory & 0.351 & 0.562 & 0.590 \\
\hline Cancer & 0.176 & 0.187 & 0 \\
\hline Unknown & 0 & 0 & 0.197 \\
\hline Other causes & 0.176 & 0 & 0 \\
\hline Patients with $\geq I \mathrm{SAE}$ & 163 & 72 & 94 \\
\hline Number of SAEs/I00 PTYs & 25.205 & 20.965 & 35.615 \\
\hline \multicolumn{4}{|c|}{ Respiratory, thoracic, and mediastinal disorders } \\
\hline Total & $84(7.377)$ & $37(6.926)$ & $61(12.003)$ \\
\hline COPD worsening & $54(4.742)$ & $28(5.24 I)$ & $48(9.445)$ \\
\hline Respiratory failure & $7(0.615)$ & $2(0.374)$ & $5(0.984)$ \\
\hline Dyspnea & $3(0.263)$ & 0 & $2(0.394)$ \\
\hline Pneumothorax & $3(0.263)$ & $2(0.374)$ & 0 \\
\hline Acute respiratory failure & $2(0.176)$ & $2(0.374)$ & 0 \\
\hline \multicolumn{4}{|l|}{ Infections and infestations } \\
\hline Total & $54(4.742)$ & $25(4.680)$ & $35(6.887)$ \\
\hline Pneumonia & $19(1.669)$ & $9(1.685)$ & $15(2.95 \mathrm{I})$ \\
\hline Bronchitis & $6(0.527)$ & $2(0.374)$ & I (0.197) \\
\hline $\begin{array}{l}\text { Upper respiratory tract } \\
\text { infection bacterial }\end{array}$ & $5(0.439)$ & $2(0.374)$ & $5(0.984)$ \\
\hline $\begin{array}{l}\text { Lower respiratory } \\
\text { tract infection }\end{array}$ & $3(0.263)$ & I (0.187) & $3(0.590)$ \\
\hline Cellulitis & $3(0.263)$ & I (0.187) & I (0.197) \\
\hline \multicolumn{4}{|l|}{ Cardiac disorders } \\
\hline Total & $34(2.986)$ & $5(0.936)$ & $16(3.148)$ \\
\hline Atrial fibrillation & $8(0.703)$ & 0 & 0 \\
\hline Acute coronary syndrome & $3(0.263)$ & 0 & 0 \\
\hline Cardiac failure congestive & $3(0.263)$ & I (0.187) & I (0.197) \\
\hline Myocardial infarction & $3(0.263)$ & I $(0.187)$ & I (0.197) \\
\hline Angina pectoris & $2(0.176)$ & I $(0.187)$ & $3(0.590)$ \\
\hline \multicolumn{4}{|l|}{ Nervous system disorders } \\
\hline Total & $16(1.405)$ & $8(1.497)$ & $6(1.181)$ \\
\hline Syncope & $6(0.527)$ & 0 & I (0.197) \\
\hline Transient ischemic attack & $4(0.35 I)$ & 0 & I (0.197) \\
\hline
\end{tabular}

Notes: Values are total number of events (events//00 PTYs) unless otherwise stated. Risk ratio values are represented as active vs placebo.

Abbreviations: COPD, chronic obstructive pulmonary disease; $\mathrm{Cl}$, confidence interval; GLY, glycopyrronium; N, patients randomized; PBO, placebo; PTYs, patient treatment years; SAEs, serious adverse events; S-db, safety database; TIO, tiotropium.

3.805 vs placebo; $95 \% \mathrm{CI}-0.875,16.550)$ and tiotropium (RR: $1.550,95 \% \mathrm{CI}-0.253,9.495$ ), exhibited an increase in the exposure-adjusted incidence of atrial fibrillation, although these were not statistically significant (Tables 7, S1 and S2). The RR for cerebrovascular events for glycopyrronium (RR: 1.95 vs placebo; $95 \%$ CI $-0.553,6.870)(95 \%$ CI of $0.553,6.870)$ was lower than that for tiotropium (RR: 2.62 
Table 7 Incidence of CCV-related AEs and FAEs (defined by SMQ) adjusted for exposure with risk ratio and $95 \% \mathrm{Cl}$ (COPD core S-db)

\begin{tabular}{|c|c|c|c|}
\hline Preferred term & $\begin{array}{l}\text { GLY } 50 \mu \mathrm{g} \\
\mathrm{N}=2,180\end{array}$ & $\begin{array}{l}\text { TIO } 18 \mu \mathrm{g} \\
\mathrm{N}=1,077\end{array}$ & $\begin{array}{l}\text { PBO } \\
\mathbf{N}=921\end{array}$ \\
\hline \multicolumn{4}{|l|}{ Cardiac arrhythmias } \\
\hline $\begin{array}{l}\text { Number (episodes/ } \\
\text { I00 PTYs) }\end{array}$ & $51(4.48)$ & $24(4.49)$ & $25(4.92)$ \\
\hline Relative risk & 0.909 & 0.770 & \\
\hline $\begin{array}{l}95 \% \mathrm{Cl} \text { (lower, upper } \\
\text { limit) }\end{array}$ & $0.562,1.472$ & $0.43 \mathrm{I}, 1.374$ & \\
\hline$P$-value & 0.6990 & 0.3758 & \\
\hline \multicolumn{4}{|c|}{ Myocardial infarction or ischemic heart disease } \\
\hline $\begin{array}{l}\text { Number (episodes/ } \\
\text { I00 PTYs) }\end{array}$ & $24(2.11)$ & $7(1.31)$ & $19(3.74)$ \\
\hline Relative risk & 0.585 & 0.352 & \\
\hline $\begin{array}{l}95 \% \mathrm{Cl} \text { (lower, upper } \\
\text { limit) }\end{array}$ & $0.320,1.071$ & $0.144,0.863$ & \\
\hline$P$-value & 0.0824 & 0.0225 & \\
\hline \multicolumn{4}{|l|}{ Atrial fibrillation } \\
\hline $\begin{array}{l}\text { Number (episodes/ } \\
\text { I00 PTYs) }\end{array}$ & $16(1.41)$ & $3(0.56)$ & $2(0.39)$ \\
\hline Relative risk & 3.805 & 1.550 & \\
\hline $\begin{array}{l}95 \% \mathrm{Cl} \text { (lower, upper } \\
\text { limit) }\end{array}$ & $0.875,16.550$ & $0.253,9.495$ & \\
\hline$P$-value & 0.0748 & 0.6354 & \\
\hline \multicolumn{4}{|l|}{ Cardiac failure } \\
\hline $\begin{array}{l}\text { Number (episodes/ } \\
\text { I00 PTYs) }\end{array}$ & II (0.97) & $2(0.37)$ & $8(1.57)$ \\
\hline Relative risk & 0.618 & 0.264 & \\
\hline $\begin{array}{l}95 \% \mathrm{Cl} \text { (lower, upper } \\
\text { limit) }\end{array}$ & $0.246,1.550$ & $0.052,1.335$ & \\
\hline$P$-value & 0.3045 & 0.1073 & \\
\hline \multicolumn{4}{|l|}{ Cerebrovascular events } \\
\hline $\begin{array}{l}\text { Number (episodes/ } \\
\text { I00 PTYs) }\end{array}$ & $13(1.14)$ & $7(|.3|)$ & $3(0.59)$ \\
\hline Relative risk & 1.950 & 2.620 & \\
\hline $\begin{array}{l}95 \% \mathrm{Cl} \text { (lower, upper } \\
\text { limit) }\end{array}$ & $0.553,6.870$ & $0.626,10.976$ & \\
\hline$P$-value & 0.2986 & 0.1875 & \\
\hline $\begin{array}{l}\text { Patients with } \geq 1 \\
\text { CCV-related FAEs }\end{array}$ & 1 & 1 & 1 \\
\hline $\begin{array}{l}\text { Number of CCV- } \\
\text { related FAEs/I00 PTYs }\end{array}$ & 0.088 & 0.187 & 0.197 \\
\hline $\begin{array}{l}\text { Cardiopulmonary } \\
\text { failure }\end{array}$ & 0 & 0 & I (0.197) \\
\hline Thalamus hemorrhage & I (0.088) & 0 & 0 \\
\hline Myocardial infarction & 0 & I (0.187) & 0 \\
\hline
\end{tabular}

Notes: CCV condition determined based on the following predefined search criteria: Cerebrovascular disorders (SMQ) (narrow); Cardiac arrhythmia terms (including bradyarrhythmias and tachyarrhythmias) (SMQ) (broad); Myocardial infarction (SMQ) (narrow); Other ischemic heart disease (SMQ) (narrow): Cardiac failure (SMQ) (narrow); Sudden death (PT); Sudden cardiac death (PT). Risk ratio values are represented as active vs placebo; Episodes $/ 100$ PTYs $=100 \times$ (n/total PTYs). Values are number of AEs/I00 PTYs (n/total PTYs) $\times 100$.

Abbreviations: AEs, adverse events; CCV, cerebrovascular and cardiovascular; $\mathrm{Cl}$, confidence interval; COPD, chronic obstructive pulmonary disease; FAEs, fatal AEs; GLY, glycopyrronium; N, patients randomized; PBO, placebo; PTYs, patient treatment years; RR, risk ratio; S-db, safety database; SMQ, Standardized MedDRA Query; TIO, tiotropium. vs placebo; 95\% CI - 0.626, 10.976) (although at wide CI). The glycopyrronium arm exhibited the least incidence of CCV-related fatal AEs (exposure-adjusted). The incidence of angioedema (exposure-adjusted and defined as Standard MedDRA Query narrow search) for glycopyrronium was similar to that for placebo and tiotropium albeit with numerically lower RR for glycopyrronium: (RR: 1.183 vs placebo; 95\% CI - 0.371, 3.773) compared with tiotropium (RR: 1.474 vs placebo; 95\% CI - 0.394, 5.519) (Table S4). Overall, the cardiovascular AE rate was similar for glycopyrronium and placebo, although atrial fibrillation events were seen more often with glycopyrronium, although not statistically significant.

\section{Long-term CCV safety (in patients with severe-to-very severe airflow limitation)}

The long-term clinical study enrolled patients at risk for exacerbations (defined as patients with severe-to-very severe airflow limitation, Stage III or IV according to GOLD 2008 criteria) and a documented history of at least one exacerbation in the previous 12 months requiring treatment with systemic corticosteroids or antibiotics, or both.

The exposure-adjusted incidence of events related to myocardial infarction, ischemic heart disease, and cardiac arrhythmia was numerically slightly higher for glycopyrronium as compared with tiotropium (Table 8); however, wide CIs preclude any clinical or statistical significance. The low number of observed cases did not allow meaningful comparison. The RR for the occurrence of cardiac failure was low for glycopyrronium (RR: 0.504 vs tiotropium; $95 \% \mathrm{CI}-0.227,1.122$ ). The incidence of cerebrovascular events (exposure-adjusted) during the long-term period was low compared to that of cardiovascular events, and also similar for tiotropium and glycopyrronium.

\section{Safety during PMS review period}

Table 9 summarizes the incidence of SAEs and non-SAEs during the PMS review phase (ie, from September 28, 2012 to March 28, 2014). By system organ class, the three most commonly occurring events during the PMS phase (in the order of decreasing frequency) were respiratory disorders, followed by gastrointestinal disorders and nervous system disorders. Cough was the most commonly occurring event across all organ system classes during the PMS review period; compared with data from clinical studies, glycopyrronium did not increase the risk of AEs and SAEs in patients during PMS. 
Table 8 Incidence of cerebrovascular and cardiovascular AEs (defined by SMQ) during long-term ( $\geq 1$ year) period and adjusted for exposure with risk ratio and 95\% CI (COPD longterm S-db)

\begin{tabular}{|c|c|c|}
\hline Preferred term & $\begin{array}{l}\text { GLY } 50 \mu \mathrm{g} \\
\mathbf{N}=\mathbf{7 4 0}\end{array}$ & $\begin{array}{l}\text { TIO I8 } \mu \mathrm{g} \\
\mathbf{N}=737\end{array}$ \\
\hline \multicolumn{3}{|l|}{ Cardiac arrhythmias } \\
\hline Number (episodes/I00 PTYs) & $29(3.45)$ & $16(1.89)$ \\
\hline Relative risk & 1.828 & \\
\hline $95 \% \mathrm{Cl}$ (lower, upper limit) & $0.993,3.365$ & \\
\hline$P$-value & 0.0528 & \\
\hline \multicolumn{3}{|c|}{ Myocardial infarction or ischemic heart disease } \\
\hline Number (episodes/I00 PTYs) & $26(3.09)$ & $19(2.24)$ \\
\hline Relative risk & 1.380 & \\
\hline $95 \% \mathrm{Cl}$ (lower, upper limit) & $0.764,2.493$ & \\
\hline$P$-value & 0.2859 & \\
\hline \multicolumn{3}{|l|}{ Atrial fibrillation } \\
\hline Number (episodes/I00 PTYs) & $14(1.66)$ & $9(1.06)$ \\
\hline Relative risk & 1.569 & \\
\hline $95 \% \mathrm{Cl}$ (lower, upper limit) & $0.679,3.624$ & \\
\hline$P$-value & 0.2919 & \\
\hline \multicolumn{3}{|l|}{ Cardiac failure } \\
\hline Number (episodes/I00 PTYs) & $9(1.07)$ & $18(2.12)$ \\
\hline Relative risk & 0.504 & \\
\hline 95\% Cl (lower, upper limit) & $0.227,1.122$ & \\
\hline$P$-value & 0.0935 & \\
\hline \multicolumn{3}{|l|}{ Cerebrovascular events } \\
\hline Number (episodes/I00 PTYs) & $9(1.07)$ & $10(1.18)$ \\
\hline Relative risk & 0.908 & \\
\hline 95\% Cl (lower, upper limit) & $0.369,2.234$ & \\
\hline$P$-value & 0.8329 & \\
\hline
\end{tabular}

Notes: CCV condition determined based on the following predefined SMQ search criteria: Angioedema (narrow scope); Cerebrovascular disorders (narrow); Cardiac arrhythmia (including bradyarrhythmias and tachyarrhythmias) (broad); Myocardial infarction (narrow); Other ischemic heart disease (narrow). Risk ratio values are represented as glycopyrronium vs tiotropium.

Abbreviations: AEs, adverse events; CCV, cerebrovascular and cardiovascular; $\mathrm{Cl}$, confidence interval; COPD, chronic obstructive pulmonary disease; GLY, glycopyrronium; N, patients randomized; PTYs, patient treatment years; RR, risk ratio; S-db, safety database; SMQ, Standardized MedDRA Query; TIO, tiotropium.

The cardiovascular safety profile of glycopyrronium was found to be consistent with the approved label as supported by data from the clinical development program. Furthermore, there was no increase in the severity or incidence of reports related to atrial fibrillation with identification of no new safety concern compared with safety information on the package insert of Seebri ${ }^{\circledR}$ Breezhaler $^{\circledR}$.

\section{Safety analysis during PMS review period using EMPIRICA ${ }^{\mathrm{TM}}$}

Based on the safety data retrieved using EMPIRICA ${ }^{\mathrm{TM}}$, EB05 disproportionality scores for all AEs were less than the threshold value $($ EB05 $<2)$ except for urinary retention and cardiac arrhythmia, which are well-known side effects of anticholinergic compounds (Table 10).
Table 9 Summary of serious and nonserious adverse reactions during PMS sorted by primary system organ class and preferred term

\begin{tabular}{|c|c|c|}
\hline $\begin{array}{l}\text { Primary system organ } \\
\text { class, preferred term }\end{array}$ & $\begin{array}{l}\text { Total } \\
\text { reports }^{\mathrm{a}}\end{array}$ & $\begin{array}{l}\text { Reporting } \\
\text { frequency }^{b}\end{array}$ \\
\hline \multicolumn{3}{|c|}{ Respiratory, thoracic, and mediastinal disorders } \\
\hline Total & 263 & 0.144 \\
\hline Cough & 59 & 0.032 \\
\hline Dyspnea & 55 & 0.030 \\
\hline Epistaxis & 25 & 0.014 \\
\hline Dysphonia & 17 & 0.009 \\
\hline Throat irritation & 17 & 0.009 \\
\hline Oropharyngeal pain & 16 & 0.009 \\
\hline \multicolumn{3}{|l|}{ Gastrointestinal disorders } \\
\hline Total & 223 & 0.122 \\
\hline Dry mouth & 38 & 0.021 \\
\hline Diarrhea & 32 & 0.018 \\
\hline Nausea & 24 & 0.013 \\
\hline Upper abdominal pain & 16 & 0.009 \\
\hline Constipation & 12 & 0.007 \\
\hline \multicolumn{3}{|l|}{ Nervous system disorders } \\
\hline Total & 106 & 0.058 \\
\hline Headache & 43 & 0.024 \\
\hline Dizziness & 13 & 0.007 \\
\hline Dysgeusia & 12 & 0.007 \\
\hline \multicolumn{3}{|c|}{ Skin and subcutaneous tissue disorders } \\
\hline Total & 81 & 0.044 \\
\hline Rash & 22 & 0.012 \\
\hline Pruritus & 11 & 0.006 \\
\hline \multicolumn{3}{|l|}{ Cardiac disorders } \\
\hline Total & 74 & 0.041 \\
\hline Palpitations & 23 & 0.013 \\
\hline Tachycardia & 13 & 0.007 \\
\hline \multicolumn{3}{|l|}{ Renal and urinary disorders } \\
\hline Total & 72 & 0.039 \\
\hline Urinary retention & 18 & 0.010 \\
\hline Dysuria & 16 & 0.009 \\
\hline Pollakiuria & 11 & 0.006 \\
\hline \multicolumn{3}{|l|}{ Musculoskeletal disorders } \\
\hline Total & 71 & 0.039 \\
\hline Myalgia & 17 & 0.009 \\
\hline Pain in extremity & 14 & 0.008 \\
\hline \multicolumn{3}{|l|}{ Eye disorders } \\
\hline Total & 66 & 0.036 \\
\hline Visual impairment & 12 & 0.007 \\
\hline \multicolumn{3}{|l|}{ Psychiatric disorders } \\
\hline Total & 44 & 0.024 \\
\hline Insomnia & 16 & 0.009 \\
\hline
\end{tabular}

Notes: ancludes serious and nonserious adverse reactions. Incidence is reported only for events that occurred with a frequency of $0.007 / 100$ PTYs.

Abbreviations: PMS, post-marketing surveillance; PTYs, patient treatment years.

\section{Discussion}

Analyzing the pooled data from clinical studies and the PMS review period of glycopyrronium offers the first opportunity for a comprehensive assessment of AEs and SAEs related to its use in COPD. The importance of this relates to the possible adverse consequences of LAMA use in this setting. 
Table 10 Statistical scores for AEs of clinical interest during the PMS phase

\begin{tabular}{ll}
\hline $\begin{array}{l}\text { Preferred term } \\
\text { (leading event) }\end{array}$ & $\begin{array}{l}\text { EB05 disproportionality } \\
\text { scores }\end{array}$ \\
\hline Angioedema (SMQ-narrow) & 0.787 \\
Angioedema (SMQ-broad) & 0.739 \\
Atrial fibrillation & 1.768 \\
Cardiac arrhythmia (nonspecific SMQ-broad) & 0.777 \\
Cardiac arrhythmia terms* (SMQ-broad) & 1.398 \\
Cardiac failure (SMQ-narrow) & 0.598 \\
Cardiac failure (SMQ-broad) & 0.417 \\
Glaucoma (SMQ-narrow) & 0.99 \\
Glaucoma (SMQ-broad) & 0.443 \\
Myocardial infarction (SMQ-narrow) & 0.223 \\
Myocardial infarction (SMQ-broad) & 0.277 \\
Urinary retention & 5.699 \\
Urinary tract disorder & 0.264
\end{tabular}

Notes: *Including bradyarrhythmias and tachyarrhythmias. EB05 disproportionality scores represent the lower $90 \% \mathrm{Cl}$ limit of Empirical Bayes Geometric Mean.

Abbreviations: AEs, adverse events; PMS, post-marketing surveillance; SMQ, Standardized MedDRA Query.

For example, cardiovascular safety concerns had been raised with the use of tiotropium in widespread usage since its launch in January 2004, evident primarily when used via the Respimat $^{\mathbb{Q}}$ device. ${ }^{3,20}$ A comprehensive examination of drug safety often continues beyond the clinical phase leading up to the PMS period, thus providing an opportunity to not only capture the occurrence of expected safety concerns but also those that are infrequent or may be unexpected. The analysis of the data from various clinical studies and the PMS review period showed that glycopyrronium did not increase the risk for any investigated safety points in comparison to placebo, although the incidence of atrial fibrillation was numerically higher with glycopyrronium vs placebo. Anticholinergics are known to be associated with cardiovascular AEs, such as arrhythmias. ${ }^{2}$ The analysis also indicates that safety profile of glycopyrronium was similar to that of tiotropium. COPD worsening was the most common $\mathrm{AE}$ and SAE reported in clinical trials, and occurred least frequently with glycopyrronium vs comparators. The long-term safety of glycopyrronium was also very similar to that of tiotropium.

There are some limitations to our analysis. In particular, clinical trials have predefined criteria for inclusion and exclusion of patients that may not always replicate the real-life setting or may not represent clinical characteristics of patients with COPD who receive the approved treatment. In addition, our reliance on incidence rates may have confounded our analysis. The incidence rate of AEs is calculated as the total number of episodes divided by the total patient years. Although this method can account for differences in exposure and appears appropriate for use when the data from patient population is pooled from studies with different exposure duration, there is an implicit assumption that each $\mathrm{AE}$ does not increase or decrease in frequency and/or severity over time. This may not be a valid assumption in all the cases. Additionally, the hygroscopic nature of tiotropium makes it challenging to remove the powder from the capsule (marked with manufacturer's logo) to a placebo capsule. In the GLOW2, SHINE (both from COPD core S-db), and SPARK (COPD long-term S-db) studies, tiotropium was dosed open-label, which may have influenced patient expectations. It is possible that in open-label studies, patients receiving the unblinded treatment may over report more favorable outcomes or, under report unfavorable safety signals. Finally, there could also be potential under reporting of AEs available in the ARGUS ${ }^{\text {TM }}$ database, which are used as background incidence for calculation.

The comprehensive analysis of safety of glycopyrronium presented here has numerous strengths since it pools data from five randomized clinical trials (from the COPD core S-db) representing more than 4,000 patients with moderateto-severe COPD. Our analysis also presents long-term safety ( $>1$ year) of glycopyrronium, specifically in patients with severe-to-very severe COPD (from the COPD long-term $\mathrm{S}-\mathrm{db}$ ), who are at an increased risk of exacerbation. ${ }^{1}$

Furthermore, the inclusion and exclusion criteria across all studies were similar and so were the clinical characteristics of recruited patients (with the exception of the SPARK study to assess long-term safety). The pooled studies also exhibit an almost identical method of collection of AE reports and allow for the analysis of cardiovascular safety in patients with COPD with different exposure duration. Along with the pooled data from clinical studies, the safety of glycopyrronium was also evaluated during the PMS review period, particularly its cardiac safety. This provides a complete picture of the safety profile of glycopyrronium. Finally, the EMPIRICA ${ }^{\mathrm{TM}}$ data mining tool allows using an innovative approach for assessing drug safety for detection of statistics of disproportionate reporting for recognizing emerging trends in spontaneous $\mathrm{AE}$ reports for effective pharmacovigilance.

\section{Conclusion}

The analysis of pooled data from various clinical studies did not reveal any increase in the overall risk for any of the investigated safety end points. Glycopyrronium exhibited a comparable safety profile to tiotropium and placebo. Furthermore, the safety of glycopyrronium during the PMS review period was consistent with its approved label and did not indicate any clinically important safety findings, indicating a favorable overall benefit-risk balance. 


\section{Acknowledgments}

We acknowledge Praveen Kaul (Novartis Healthcare Pvt., Ltd., India) for medical writing support. We also thank all the clinical investigators and study coordinators at the participating centers and all the patients who participated in the studies. No restrictions were placed on the authors regarding the statements made in the manuscript. This work was funded by Novartis Pharmaceuticals. The funding source was involved in the study design, the collection, management, analysis, and interpretation of the data, and in the preparation, review, and approval of the manuscript.

\section{Author contributions}

All authors contributed toward data analysis, drafting and revising the paper and agree to be accountable for all aspects of the work.

\section{Disclosure}

ADD has received research, consulting, and lecturing fees from GlaxoSmithKline, Sepracor, Schering-Plough, Altana, Methapharm, AstraZeneca, ONO Pharma, Merck Canada, Forest Laboratories, Novartis Canada/USA, Boehringer Ingelheim (Canada) Ltd., Pfizer Canada, SkyePharma, and KOS Pharmaceuticals.

EMK has served on advisory boards, speaker panels, or received travel reimbursement for Amphastar, AstraZeneca (Pearl Pharma), Boehringer-Ingelheim, Forest, GlaxoSmithKline, Merck (Schering-Plough), Mylan, Novartis, Sanofi Aventis, Sunovion, Teva and Theravance. He has conducted multicenter clinical research trials for approximately forty pharmaceutical companies.

$\mathrm{KRC}$ in the past 3 years has received compensation for consulting with Boehringer Ingelheim, CSL Behring, GlaxoSmithKline, Merck Frosst, Novartis, Takeda, Pfizer, Roche, Schering-Plough, and Grifols; has undertaken research funded by AstraZeneca, Boehringer Ingelheim, CSL Behring, Forest Laboratories, GlaxoSmithKline, Novartis, Parangenix, Roche, Takeda, and Grifols; and has participated in continuing medical education activities sponsored in whole or in part by AstraZeneca, Boehringer Ingelheim, GlaxoSmithKline, Grifols, Merck Frosst, Novartis, Takeda, and Pfizer. He is participating in research funded by the Canadian Institutes of Health Research operating grant entitled: Canadian Cohort Obstructive Lung Disease (http://clinicaltrials.gov NCT00920348). Professor Chapman holds the GSK-CIHR Research Chair in Respiratory Health Care Delivery at the University Health Network, Toronto, Canada.

MD has been part of the Advisory Board for Boehringer-Pfizer, GSK, Nycomed, and Altana. He has performed consulting work for Boehringer-Pfizer, GSK, AstraZeneca, and Dompé. He also received lecture fees from these companies. All of the above amounted to less than 10,000 euro/year. He received a research grant of 45,000 euro/year from AstraZeneca. PDA, HH, and PA are employees of Novartis Pharmaceuticals Corporation. RDG and PG are employees of Novartis Pharma AG. The authors report no other conflicts of interest in this work.

\section{References}

1. Global Strategy for the Diagnosis, Management and Prevention of COPD. Global Initiative for Chronic Obstructive Lung Disease (GOLD); 2015. Available from: http://www.goldcopd.org/. Accessed April 22, 2015.

2. Sharafkhaneh A, Majid H, Gross NJ. Safety and tolerability of inhalational anticholinergics in COPD. Drug Healthc Patient Saf. 2013;5: 49-55.

3. Singh S, Loke YK, Enright PL, Furberg CD. Mortality associated with tiotropium mist inhaler in patients with chronic obstructive pulmonary disease: systematic review and meta-analysis of randomised controlled trials. BMJ. 2011;2011(342):d3215.

4. Wise RA, Anzueto A, Cotton D, et al; IOSPIR Investigators. Tiotropium respimat inhaler and the risk of death in COPD. N Engl J Med. 2013; 369(16):1491-1501.

5. Jenkins CR. More than just reassurance on tiotropium safety. $N$ Engl J Med. 2013;369(16):1555-1556.

6. Jenkins CR. Tiotropium and the risk of death in COPD. N Engl J Med. 2014;370(5):482-483.

7. Loke YK, Singh S, Furberg CD. Tiotropium and the risk of death in COPD. N Engl J Med. 2014;30(5):480-481.

8. Verhamme KM, van Blijderveen N, Sturkenboom MC. Tiotropium and the risk of death in COPD. N Engl J Med. 2014;370(5):481-482.

9. Wise RA. Tiotropium and the risk of death in COPD. $N$ Engl J Med. 2014;370(5):482.

10. Fogarty C, Hattersley H, Di SL, Drollmann A. Bronchodilatory effects of NVA237, a once daily long-acting muscarinic antagonist, in COPD patients. Respir Med. 2011;105(3):337-342.

11. D'Urzo AD, Kerwin E, Overend T, D'Andrea P, Chen H, Goyal P. Once daily glycopyrronium for the treatment of COPD: pooled analysis of the GLOW1 and GLOW2 studies. Curr Med Res Opin. 2014;30(3): 493-508.

12. Sykes DA, Dowling MR, Leighton-Davies J, et al. The Influence of receptor kinetics on the onset and duration of action and the therapeutic index of NVA237 and tiotropium. J Pharmacol Exp Ther. 2012;343(2): 520-528.

13. D’Urzo AD, Ferguson GT, van Noord JA, et al. Efficacy and safety of once-daily NVA237 in patients with moderate-to-severe COPD: the GLOW1 trial. Respir Res. 2011;2011(12):156.

14. Kerwin E, Hébert J, Gallagher N, et al. Efficacy and safety of NVA237 versus placebo and tiotropium in patients with COPD: the GLOW2 study. Eur Respir J. 2012;40(5):1106-1114.

15. Bateman ED, Ferguson GT, Barnes N, et al. Dual bronchodilation with QVA149 versus single bronchodilator therapy: the SHINE study. Eur Respir J. 2013;42(6):1484-1494.

16. Chapman KR, Beeh KM, Beier J, et al. A blinded evaluation of the efficacy and safety of glycopyrronium, a once-daily long-acting muscarinic antagonist, versus tiotropium, in patients with COPD: the GLOW5 study. BMC Pulm Med. 2014;2014(14):4.

17. Chen W, Tieying S, Yijiang H, et al. Efficacy and safety of once-daily glycopyrronium in predominantly Chinese patients with moderate-tosevere chronic obstructive pulmonary disease (COPD): the GLOW7 study. B104. It's No Longer a Secret Service: Updates in COPD Pharmacotherapy. American Thoracic Society, San Diego, USA 2014: A3756-A3756. 
18. Wedzicha JA, Decramer M, Ficker JH, et al. Analysis of chronic obstructive pulmonary disease exacerbations with the dual bronchodilator QVA149 compared with glycopyrronium and tiotropium (SPARK): a randomised, double-blind, parallel-group study. Lancet Respir Med. 2013;1(3):199-209.

19. Szarfman A, Machado SG, O’Neill RT. Use of screening algorithms and computer systems to efficiently signal higher-than-expected combinations of drugs and events in the US FDA's spontaneous reports database. Drug Saf. 2002;25(6):381-392.
20. Singh S, Loke YK, Furberg CD. Inhaled anticholinergics and risk of major adverse cardiovascular events in patients with chronic obstructive pulmonary disease: a systematic review and meta-analysis. JAMA. 2008; 300(12):1439-1450. 


\section{Supplementary materials}

Table SI Number of patients with atrial fibrillation and flutter (adjudicated) adjusted for exposure (COPD core S-db)

\begin{tabular}{|c|c|c|c|}
\hline & GLY $50 \mu \mathrm{g}$ & TIO $18 \mu \mathrm{g}$ & PBO \\
\hline \multicolumn{4}{|l|}{ Overall } \\
\hline Total population & 2,180 & I,077 & 921 \\
\hline Patients with $\geq I$ event & 44 & 23 & 14 \\
\hline Total patient years & I, I 38.642 & 534.234 & 508.216 \\
\hline Patients/I00 PTYs & 3.864 & 4.305 & 2.755 \\
\hline \multicolumn{4}{|l|}{ New onset } \\
\hline Total population & 2,124 & 1,049 & 900 \\
\hline Patients with $\geq I$ event & 10 & 5 & I \\
\hline Total patient years & I,III.373 & 519.661 & 498.042 \\
\hline Patients/I00 PTYs & 0.900 & 0.962 & 0.201 \\
\hline \multicolumn{4}{|l|}{ Recurrent } \\
\hline Total population & 56 & 28 & 21 \\
\hline Patients with $\geq I$ event & 34 & 18 & 13 \\
\hline Total patient years & 27.269 & 14.574 & 10.174 \\
\hline Patients/I00 PTYs & I 24.684 & $|23.5| \mid$ & | 27.779 \\
\hline
\end{tabular}

Abbreviations: COPD, chronic obstructive pulmonary disease; GLY, glycopyrronium; PBO, placebo; PTYs, patient treatment years; S-db, safety database; TIO, tiotropium.

Table S2 Number of patients with atrial fibrillation and flutter (adjudicated) adjusted for exposure for long-term safety assessment (COPD long-term S-db)

\begin{tabular}{lll}
\hline & GLY $\mathbf{5 0} \mu \mathbf{g}$ & TIO I8 $\boldsymbol{\mu g}$ \\
\hline Overall & & 737 \\
Total population & 740 & 24 \\
Patients with $\geq I$ event & 21 & 848.780 \\
Total patient years & 841.645 & 2.828 \\
Patients/I00 PTYs & 2.495 & 714 \\
New onset & & 8 \\
Total population & 717 & 825.944 \\
Patients with $\geq I$ event & 6 & 0.969 \\
Total patient years & 817.615 & \\
Patients/I00 PTYs & 0.734 & 23 \\
Recurrent & & 16 \\
Total population & 23 & 22.836 \\
Patients with $\geq I$ event & 15 & 70.064 \\
Total patient years & 24.030 & \\
Patients/I00 PTYs & 62.422 & \\
\hline
\end{tabular}

Abbreviations: COPD, chronic obstructive pulmonary disease; GLY, glycopyrronium; PTYs, patient treatment years; S-db, safety database; TIO, tiotropium.

Table S3 Incidence of CCV-related AEs (per 100 patient years) in clinical studies sorted by primary system organ class and preferred term ( $>10$ events for GLY) (COPD core S-db)

\begin{tabular}{|c|c|c|c|}
\hline $\begin{array}{l}\text { Primary system organ class, } \\
\text { preferred term }\end{array}$ & $\begin{array}{l}\text { GLY } 50 \mu \mathrm{g} \\
\mathrm{N}=2,180\end{array}$ & $\begin{array}{l}\text { TIO } 18 \mu \mathrm{g} \\
\mathrm{N}=1,077\end{array}$ & $\begin{array}{l}\text { PBO } \\
\mathbf{N}=92 \text { I }\end{array}$ \\
\hline Patients with $\geq I A E$ & 83 & 31 & 37 \\
\hline Number of AEs/I00 PTYs & 8.695 & 7.487 & 10.822 \\
\hline \multicolumn{4}{|l|}{ Cardiac disorders } \\
\hline Total & $83(7.289)$ & $27(5.054)$ & $44(8.658)$ \\
\hline Atrial fibrillation & $15(1.317)$ & $4(0.749)$ & $2(0.394)$ \\
\hline Angina pectoris & $10(0.878)$ & $5(0.936)$ & $10(1.968)$ \\
\hline Cardiac failure congestive & $6(0.527)$ & I (0.187) & $2(0.394)$ \\
\hline Ventricular extrasystoles & $6(0.527)$ & $4(0.749)$ & $2(0.394)$ \\
\hline
\end{tabular}


Table S3 (Continued)

\begin{tabular}{|c|c|c|c|}
\hline & $\begin{array}{l}\text { GLY } 50 \mu \mathrm{g} \\
\mathrm{N}=2,180\end{array}$ & $\begin{array}{l}\text { TIO } 18 \mu \mathrm{g} \\
\mathrm{N}=1,077\end{array}$ & $\begin{array}{l}\text { PBO } \\
\mathrm{N}=921\end{array}$ \\
\hline Atrioventricular block first-degree & $4(0.35 I)$ & $\mathrm{I}(0.187)$ & $2(0.394)$ \\
\hline Acute coronary syndrome & $3(0.263)$ & 0 & 0 \\
\hline Cardiac failure & $3(0.263)$ & I (0.187) & 0 \\
\hline Coronary artery disease & $3(0.263)$ & 0 & $2(0.394)$ \\
\hline Myocardial infarction & $3(0.263)$ & I (0.187) & $2(0.394)$ \\
\hline Myocardial ischemia & $3(0.263)$ & 0 & I (0.197) \\
\hline Supraventricular extrasystoles & $3(0.263)$ & $3(0.562)$ & $3(0.590)$ \\
\hline Supraventricular tachycardia & $3(0.263)$ & I $(0.187)$ & $4(0.787)$ \\
\hline Atrial flutter & $2(0.176)$ & 0 & $4(0.787)$ \\
\hline Bundle branch block left & $2(0.176)$ & $2(0.374)$ & 0 \\
\hline Bundle branch block right & $2(0.176)$ & 0 & 0 \\
\hline Sinus tachycardia & $2(0.176)$ & $2(0.374)$ & $2(0.394)$ \\
\hline Acute myocardial infarction & I (0.088) & 0 & I (0.197) \\
\hline Arteriosclerosis coronary artery & I (0.088) & 0 & I (0.197) \\
\hline Atrial tachycardia & I $(0.088)$ & 0 & 0 \\
\hline Atrioventricular block & I (0.088) & 0 & 0 \\
\hline Atrioventricular dissociation & I (0.088) & 0 & 0 \\
\hline Cor pulmonale & I (0.088) & 0 & $2(0.394)$ \\
\hline Cor pulmonale chronic & I (0.088) & 0 & 0 \\
\hline Long QT syndrome & I (0.088) & 0 & 0 \\
\hline Nodal arrhythmia & I (0.088) & 0 & 0 \\
\hline Sick sinus syndrome & I (0.088) & 0 & 0 \\
\hline Sinus bradycardia & I (0.088) & 0 & I (0.197) \\
\hline Tachyarrhythmia & I (0.088) & 0 & I $(0.197)$ \\
\hline Wandering pacemaker & I (0.088) & 0 & 0 \\
\hline Angina unstable & 0 & 0 & I (0.197) \\
\hline Arrhythmia & 0 & I (0.I87) & 0 \\
\hline Cardiac failure acute & 0 & 0 & I $(0.197)$ \\
\hline Cardiopulmonary failure & 0 & 0 & I (0.197) \\
\hline Coronary artery insufficiency & 0 & I (0.I87) & 0 \\
\hline Right ventricular failure & 0 & 0 & I (0.197) \\
\hline Ventricular tachycardia & 0 & 0 & 0 \\
\hline \multicolumn{4}{|l|}{ Nervous system disorders } \\
\hline Total & $13(1.142)$ & $6(1.123)$ & $3(0.590)$ \\
\hline Transient ischemic attack & $5(0.439)$ & 0 & $2(0.394)$ \\
\hline Carotid artery stenosis & $2(0.176)$ & 0 & 0 \\
\hline Carotid artery disease & I (0.088) & 0 & 0 \\
\hline Cerebral arteriosclerosis & I (0.088) & 0 & 0 \\
\hline Cerebral infarction & I (0.088) & 0 & 0 \\
\hline Cerebrovascular accident & I (0.088) & $2(0.374)$ & 0 \\
\hline Thalamus hemorrhage & I (0.088) & 0 & 0 \\
\hline Vertebrobasilar insufficiency & I (0.088) & 0 & I (0.197) \\
\hline Hemorrhagic stroke & 0 & I (0.187) & 0 \\
\hline Ischemic stroke & 0 & $2(0.374)$ & 0 \\
\hline Spinal hematoma & 0 & I (0.187) & 0 \\
\hline \multicolumn{4}{|c|}{ Respiratory, thoracic, and mediastinal disorders } \\
\hline Total & 0 & 0 & I (0.197) \\
\hline Pulmonary edema & 0 & 0 & I (0.197) \\
\hline \multicolumn{4}{|c|}{ Injury, poisoning, and procedural complications } \\
\hline Total & 0 & I (0.187) & 0 \\
\hline Extradural hematoma & 0 & I (0.187) & 0 \\
\hline \multicolumn{4}{|l|}{ Investigations } \\
\hline Total & $3(0.263)$ & $6(1.123)$ & $7(1.377)$ \\
\hline Heart rate irregular & $2(0.176)$ & 0 & 0 \\
\hline Electrocardiogram QT prolonged & I (0.088) & $6(1.123)$ & $7(1.377)$ \\
\hline
\end{tabular}

Note: Values in parentheses are total number of AEs per 100 PTYs.

Abbreviations: AEs, adverse events; CCV, cerebrovascular and cardiovascular; COPD, chronic obstructive pulmonary disease; GLY, glycopyrronium; N, patients randomized; PBO, placebo; PTYs, patient treatment years; S-db, safety database; TIO, tiotropium. 
Table S4 Incidence of angioedema (defined by SMQ)-narrow AE episodes adjusted for exposure by primary system organ class and preferred term with RR and $95 \% \mathrm{Cl}$ (COPD core S-db)

\begin{tabular}{|c|c|c|c|}
\hline & $\begin{array}{l}\text { GLY } 50 \mu \mathrm{g} \\
\mathrm{N}=2,180\end{array}$ & $\begin{array}{l}\text { TIO I8 } \mu \mathrm{g} \\
\mathrm{N}=1,077\end{array}$ & $\begin{array}{l}\text { PBO } \\
\mathbf{N}=92 \text { I }\end{array}$ \\
\hline Patients with $\geq I$ event & 9 & 4 & 4 \\
\hline Episodes/I00 PTYs & 0.878 & 1.123 & 0.787 \\
\hline Risk ratio & 1.183 & 1.474 & \\
\hline 95\% Cl (lower, upper limit) & $0.371,3.773$ & $0.394,5.519$ & \\
\hline$P$-value & 0.7762 & 0.5643 & \\
\hline \multicolumn{4}{|l|}{ Eye disorders } \\
\hline Total & I (0.088) & 0 & I $(0.197)$ \\
\hline Periorbital edema & I (0.088) & 0 & 0 \\
\hline Eye swelling & 0 & 0 & I $(0.197)$ \\
\hline \multicolumn{4}{|l|}{ Gastrointestinal disorders } \\
\hline Total & 0 & $2(0.374)$ & I $(0.197)$ \\
\hline Lip edema & 0 & $2(0.374)$ & 0 \\
\hline Palatal edema & 0 & 0 & I $(0.197)$ \\
\hline \multicolumn{4}{|c|}{ Skin and subcutaneous tissue disorders } \\
\hline Total & $9(0.790)$ & $4(0.749)$ & $2(0.394)$ \\
\hline Urticaria & $9(0.790)$ & $3(0.562)$ & $2(0.394)$ \\
\hline Angioedema & 0 & I (0.187) & 0 \\
\hline
\end{tabular}

Notes: Risk ratio values are represented as active vs placebo. Values in parentheses are total number of AEs per 100 PTYs.

Abbreviations: AE, adverse event; $\mathrm{Cl}$, confidence interval; COPD, chronic obstructive pulmonary disease; GLY, glycopyrronium; N, patients randomized; PBO, placebo; PTYs, patient treatment years; RR, risk ratio; S-db, safety database; SMQ, Standardized MedDRA Query; TIO, tiotropium.

\section{Publish your work in this journal}

The International Journal of COPD is an international, peer-reviewed journal of therapeutics and pharmacology focusing on concise rapid reporting of clinical studies and reviews in COPD. Special focus is given to the pathophysiological processes underlying the disease, intervention programs, patient focused education, and self management protocols.

\section{Dovepress}

This journal is indexed on PubMed Central, MedLine and CAS. The manuscript management system is completely online and includes a very quick and fair peer-review system, which is all easy to use. Visit $\mathrm{http} / / / \mathrm{www}$.dovepress.com/testimonials.php to read real quotes from published authors. 